\title{
Prophylactic antibiotic regimens in tumor surgery (PARITY) survey
}

Khaled Hasan ${ }^{1 *}$, Antonella Racano ${ }^{1}$, Benjamin Deheshi ${ }^{1}$, Forough Farrokhyar ${ }^{1,4}$, Jay Wunder ${ }^{2}$, Peter Ferguson ${ }^{2}$, Ginger Holt ${ }^{3}$, Herbert Schwartz ${ }^{3}$, Brad Petrisor ${ }^{1}$, Mohit Bhandari ${ }^{1}$ and Michelle Ghert ${ }^{1}$

\begin{abstract}
Background: Deep infection following endoprosthetic limb reconstruction for sarcoma of the long bones is a devastating complication occurring in 15\% of sarcoma patients. Optimizing infection protocols and conducting definitive surgical trials are critical to improving outcomes. In this study, the PARITY (Prophylactic Antibiotic Regimens in Tumor Surgery) investigators aimed to examine surgeon preferences in antibiotic prophylaxis and perceptions about current evidence, as well as to ascertain interest in resolving uncertainty in the evidence with clinical trials.

Methods: We used a cross-sectional survey to examine current practice in the prescription of prophylactic antibiotics in Musculoskeletal Tumor Surgery. The survey was approved by our institution's Ethics Board and emailed to all Active Members of the Musculoskeletal Tumor Society (MSTS) and Canadian Orthopaedic Oncology Society (CANOOS). Survey answers were collected using an anonymous online survey tool.

Results: Of the 96 surgeons who received the questionnaire, 72 responded (75\% response rate (\% Cl: $65.5,82.5 \%)$ ). While almost all respondents agreed antibiotic regimens were important in reducing the risk of infection, respondents varied considerably in their choices of antibiotic regimens and dosages. Although $73 \%$ (95\% Cl: 61, $82 \%$ ) of respondents prescribe a first generation cephalosporin, $25 \%$ favor additional coverage with an aminoglycoside and/or Vancomycin. Of those who prescribe a cephalosporin, 33\% prescribe a dosage of one gram for all patients and the reminder prescribe up to 2 grams based on body weight. One in three surgeons (95\% Cl: 25, 48\%) believes antibiotics could be discontinued after 24 hours but 40\% (95\% Cl: 30, 53\%) continue antibiotics until the suction drain is removed. Given the ongoing uncertainty in evidence to guide best practices, 90\% (95\% Cl: $81,95 \%)$ of respondents agreed that they would change their practice if a large randomized controlled trial showed clear benefit of an antibiotic drug regimen different from what they are currently using. Further support for a clinical trial was observed by an overwhelming surgeon interest (87\%; 95\% Cl: 77, 93\%) in participating in a multi-center randomized controlled study.
\end{abstract}

Conclusion: The current lack of guidelines for the prescription of prophylactic antibiotics in Musculoskeletal Tumor Surgery has left Orthopaedic Oncologists with varying opinions and practices. The lack of current evidence and strong surgeon support for participating in a definitive study provides strong rationale for clinical trials.

\footnotetext{
* Correspondence: khaled.hasan@medportal.ca

'Department of Surgery, McMaster University, Hamilton, ON, Canada

Full list of author information is available at the end of the article
}

\section{Biomed Central}

(c) 2012 Hasan et al.; licensee BioMed Central Ltd. This is an Open Access article distributed under the terms of the Creative Commons Attribution License (http://creativecommons.org/licenses/by/2.0), which permits unrestricted use, distribution, and reproduction in any medium, provided the original work is properly cited. 


\section{Background}

Limb salvage surgery is the standard of care for the vast majority of patients with long-bone sarcoma such as Ewing's Sarcoma and Osteosarcoma [1]. Limb-salvage reconstruction techniques generally involve replacement of the affected bone segment and joint with a prosthetic implant designed to recreate the normal anatomy. Preservation of neurovascular structures and the need to avoid tumor contamination of the wound result in lengthy, complicated procedures. In addition, many patients require pre- and post-operative chemotherapy for systemic disease management. These factors result in a high incidence of deep post-operative infections $(15 \%-30 \%)$ requiring surgical intervention and eventual limb-salvage failure (amputation) in approximately $50 \%$ of cases [2-6].

The high infection rates have been stable over the past 2 decades [2,3,5,7]. The standard guidelines for antibiotic prophylaxis as dictated by the American Academy of Orthopaedic Surgeons (AAOS) for total joint replacements includes a pre-operative dose of antibiotics with gram-positive coverage followed by 24 hours of postoperative antibiotics $[7,8]$. In fact, the only dose that has been shown to be important in preventing post-operative infections is the pre-operative dose $[7,9,10]$.

In higher-risk cases of tumor prosthesis surgery, no guidelines exist to direct antibiotic management. In fact, many tumor surgeons prescribe several days of antibiotics and add gram-negative coverage. However, there is no data to support these practices and the issue of antibiotic resistance with over-prescription therefore becomes important. There has been a clear link between antibiotic overprescription and the emergence of resistant microbial organisms $[8,9,11]$.

To explore current practice in the regimens of antibiotic prophylaxis used for tumor surgery, we conducted an international survey of practicing orthopaedic oncology surgeons in order to learn about their preferences with regards to this practice and to identify the need for future research in this area. Furthermore, we reasoned that the results of this survey may identify factors that influence a surgeon's preference for a particular regimen, serve to educate the orthopaedic community on issues regarding prosthesis infection, and allow for the development of future clinically related trials, which could help develop an international guideline for prophylactic antibiotic regimens in musculoskeletal tumor surgery.

\section{Methods}

\section{Question development Item generation}

We developed a questionnaire using focus groups, key informants, and the previous literature.

The items generated from the focus group were improved by data from a MEDLINE search of articles published from 1975 to 2011 using text words "infection," "sarcoma," "surgery," and "antibacterial agents". Further items were generated with key informants. Surgeons specializing in orthopaedic oncology provided additional input into potential items for the questionnaire.

\section{Pretesting and validity assessments}

In order to accurately address the need for antibiotic prophylaxis guidelines in tumour prosthesis surgery, the questionnaire was pretested amongst an independent group of four orthopaedic oncologists (face validity) with respect to reconstruction type, antibiotics used, and time points and dosages administered (content validity). These surgeons also commented on the clarity and comprehensiveness of the questionnaire.

The questionnaire itself consisted four sections, and presented closed-ended questions as multiple-choice or five-point Likert scale formats. Section A encompasses

Table 1 Physician demographics

\begin{tabular}{|c|c|c|c|}
\hline Characteristic & & No. & (\%) \\
\hline \multirow[t]{5}{*}{ Age } & Less than 30 & 0 & $(0 \%)$ \\
\hline & $30-40$ & 12 & $(16.7 \%)$ \\
\hline & $41-50$ & 25 & $(34.7 \%)$ \\
\hline & $51-60$ & 27 & $(37.5 \%)$ \\
\hline & Over 60 & 8 & $(11.1 \%)$ \\
\hline \multirow[t]{5}{*}{ Number of years in practice } & Less than 5 & 3 & $(4.2 \%)$ \\
\hline & $05-10$ & 20 & $(28.2 \%)$ \\
\hline & $11-15$ & 9 & $(12.7 \%)$ \\
\hline & $16-20$ & 13 & $(18.3 \%)$ \\
\hline & Over 20 & 26 & $(36.6 \%)$ \\
\hline \multirow[t]{2}{*}{ Type of Hospital } & Academic & 62 & $(86.1 \%)$ \\
\hline & Non-Academic & 10 & $(13.9 \%)$ \\
\hline \multirow[t]{2}{*}{ Supervise residents in training } & Yes & 65 & $(90.3 \%)$ \\
\hline & No & 7 & $(9.7 \%)$ \\
\hline \multirow{2}{*}{$\begin{array}{l}\text { Completed a fellowship } \\
\text { in Orthopaedic Oncology }\end{array}$} & Yes & 69 & $(97.2 \%)$ \\
\hline & No & 2 & $(2.8 \%)$ \\
\hline \multirow{4}{*}{$\begin{array}{l}\text { Proportion of practice with } \\
\text { bone or soft-tissue tumors }\end{array}$} & $0-25 \%$ & 5 & $(5.6 \%)$ \\
\hline & $26-50 \%$ & 17 & $(23.6 \%)$ \\
\hline & $51-75 \%$ & 12 & $(16.7 \%)$ \\
\hline & $76-100 \%$ & 39 & $(54.2 \%)$ \\
\hline \multirow{7}{*}{$\begin{array}{l}\text { Number of long bone } \\
\text { sarcomas treated per year }\end{array}$} & 0 & 0 & $(0 \%)$ \\
\hline & $1-5$ & 8 & $(11.4 \%)$ \\
\hline & $6-10$ & 12 & $(17.1 \%)$ \\
\hline & $11-15$ & 12 & $(17.1 \%)$ \\
\hline & $16-20$ & 16 & $(22.9 \%)$ \\
\hline & $21-25$ & 9 & $(12.9 \%)$ \\
\hline & $>25$ & 13 & $(18.6 \%)$ \\
\hline
\end{tabular}


Table 2 Effectiveness of antimicrobial coverage relative to gram positive coverage alone in reducing infection risk in long-bone reconstruction

\begin{tabular}{llllll}
\hline Antimicrobial Coverage & $\begin{array}{l}\text { Definitely less } \\
\text { effective than } \\
\text { Gram +alone }\end{array}$ & $\begin{array}{l}\text { Moderately less } \\
\text { effective than } \\
\text { Gram + alone }\end{array}$ & $\begin{array}{l}\text { Equivalentto } \\
\text { Gram + alone }\end{array}$ & $\begin{array}{l}\text { Moderately more } \\
\text { effective than } \\
\text { Gram + alone }\end{array}$ & $\begin{array}{l}\text { Definitely more } \\
\text { effective than } \\
\text { Gram + alone }\end{array}$ \\
\hline Gram + and Gram - coverage & $2(3.0 \%)$ & $2(3.0 \%)$ & $33(50.0 \%)$ & $23(34.8 \%)$ & $6(9.1 \%)$ \\
\hline Gram - coverage alone & $40(60.6 \%)$ & $16(24.2 \%)$ & $10(15.2 \%)$ & $0(0.0 \%)$ & $0(0.0 \%)$ \\
\hline Vancomycin & $3(4.6 \%)$ & $6(9.2 \%)$ & $31(47.7 \%)$ & $22(33.8 \%)$ & $3(4.6 \%)$ \\
\hline
\end{tabular}

eight questions relating to surgeons' backgrounds (i.e. age, gender, years in practice, type of practice [academic versus community setting], fellowship training in orthopaedic oncology, and supervision of resident trainees) and surgical volume. Section B sought information regarding surgeons' management of oncology cases - specifically with regards to how long after chemotherapy can surgery be safely performed, at what white blood cell count the patient is safe for surgery. Section $C$ consists of nine questions that address features of a particular surgeon's antibiotic regimen (i.e. reconstruction type, specific antibiotics used, time period [pre- and/or post-operatively] and dosages administered). Section D addresses the need for future research in this area and provides participants with an opportunity to offer comments and/or suggestions.

\section{Questionnaire administration}

All Active Members of the Musculoskeletal Tumor Society (MSTS) and all members of the Canadian Orthopaedic Oncology Society (CANOOS) (duplicates were excluded) were surveyed voluntarily via a web-based method (Survey Monkey). Potential participants were sent the survey by a party independent of the study's investigators, with no monetary incentives. One e-mail pre-notification was provided. All response data was collected anonymously and grouped according to predefined analyses. The Ethics Review Board in conjunction with Hamilton Health Sciences approved this study. No monetary incentives or pre-notification telephone calls were used for this survey. Individual responses were kept confidential and questionnaire completion was voluntary.

\section{Sample size}

We had a response rate goal of $70 \%$ to ensure the results would be adequately powered to prevent the biased ascertainment of outcomes due to non-responder bias [12-
14]. To determine the number of respondents needed to sufficiently power our analysis, we assumed that approximately $40 \%$ of surgeons surveyed used prophylactic antibiotics until the suction drain is removed for longbone reconstruction postoperatively. Using the following formula:

$$
\begin{aligned}
& N=\left(z ^ { 2 } * \left(p(1-p) / w^{2}\right.\right. \\
& N=\left(1.96^{2} * 0.4 * 0.6\right) / 0.05^{2} \\
& N=368.8
\end{aligned}
$$

Where:

$\mathbf{N}$ = required sample size

$\mathrm{Z}=\mathrm{z}$ value (1.96 for $95 \%$ confidence interval)

$\mathbf{w}=$ the confidence interval, expressed as

decimal $(0.05=+/-5)$

$\mathbf{p}=$ percentage picking a choice (until suction drain removed), expressed in decimal $(40 \%=0.40)$

It was calculated that 369 completed questionnaires would be required to produce a $95 \%$ confidence interval (CI) of $+/-5 \%$ around the percentages of postoperative prescription of prophylactic antibiotics until suction drain is removed, with an alpha level of 0.05. A total of 96 surgeons were approached to participate. The response rate was $75 \%$ (95\% CI: 66\%, 84\%). The sample size of 72 completed survey allowed a $95 \%$ CI of $+/-12 \%$ around the percentages of the use prophylactic antibiotic until suction drain is removed for long-bone reconstruction postoperatively.

\section{Statistical analysis}

A previous report has shown that closed-ended questions result in fewer incomplete questionnaires than open-ended formats [15]. The current questionnaire framed the response options in one of two ways: fivepoint Likert scales or nominal scales. The proportion of

Table 3 Length of time prophylactic antibiotics prescribed following long-bone reconstruction

\begin{tabular}{lllll}
\hline Type of Reconstruction & $\mathbf{2 4}$ hours & $\mathbf{4 8}$ hours & $\mathbf{3 - 7}$ days & Until suction drain is removed \\
\hline Tumor prosthesis & $25(35.7 \%)$ & $13(18.6 \%)$ & $3(4.3 \%)$ & $29(41.4 \%)$ \\
\hline Allograft & $18(26.5 \%)$ & $13(19.1 \%)$ & $11(16.2 \%)$ & $26(38.2 \%)$ \\
\hline Allograft-prosthesis composite & $17(25.4 \%)$ & $12(17.9 \%)$ & $9(13.4 \%)$ & $29(43.3 \%)$ \\
\hline
\end{tabular}


participants for each multiple-choice answer with 95\% confidence intervals (CI) using Wilson's exact method were calculated.

\section{Results}

Characteristics of the respondents

Of the 96 surgeons who received the questionnaire, 72 (75\%; 95\% CI: 65.5, 82.5\%) responded. The typical respondent was a surgeon over 40 yrs old with over 5 yrs in practice (Table 1 ). The majority $(86.1 \%)$ work in an academic centre and supervise trainees (90\%)] (Table 1). Ninety-seven percent of respondents had completed further orthopaedic oncology fellowship training and 70\% of respondents spend greater than $50 \%$ of their practice treating orthopaedic oncology patients (Table 1).

\section{Management preferences}

Over $90 \%$ (95\% CI: 90, 99\%) of respondents believe that prophylactic antibiotic protocols are important to decrease the risk of infection in all long bone sarcoma reconstruction types. Forty-six percent (95\% CI: 35, 57\%) believe that preoperative antibiotic administration is the single most important initial step to preventing postoperative infection while 50\% (95\% CI: 38, 61\%) believe that both preoperative and post-operative antibiotic administration are important to prevent postoperative infection.

\section{Antibiotic regimens}

Seventy-three percent (95\% CI: 61, 82\%) of respondents routinely prescribe gram-positive coverage alone for long bone reconstruction while $11 \%$ prescribe gram positive and gram negative coverage. Four percent (95\% CI: 1, $12 \%)$ of respondents prescribe Vancomycin alone. When comparing regimens to gram positive coverage alone, $50 \%$ (95\% CI: 38, 62\%) responded it would be equivalent to a combination of gram positive and gram negative prophylaxis (Table 2).

\section{Duration of antibiotics}

Thirty-six percent (95\% CI: 25, 48\%) of respondents practice discontinuing antibiotics after $24 \mathrm{hrs}$ and $18 \%$ (95\% CI:11, 29\%) discontinue at 48 hours. However $41 \%$ (95\% CI: 30, 53\%) continue antibiotics until the suction drain is removed (Table 3). Forty-three percent (95\% CI: $32,55 \%$ ) of respondents believe that there is currently no evidence to guide surgeons regarding the optimal antibiotic prophylaxis in long-bone reconstruction.

\section{First generation cephalosporin dosing}

Thirteen percent (95\% CI: 7, 23\%) of surgeons responded to prescribing one gram of Ancef (Cefazolin) per dose, while 33\% (95\% CI: 23, 44\%) prescribe two grams of Ancef per dose (Table 4). Another 53\% (95\%
Table 4 Dosage of Ancef (first generation cephalosporin) prescribed (if applicable)

\begin{tabular}{ll}
\hline Dosage & No. (\%) \\
\hline $\mathbf{1 ~ g}$ & $9(12.9 \%)$ \\
\hline $\mathbf{2} \mathbf{g}$ & $23(32.9 \%)$ \\
\hline $\mathbf{2} \mathbf{g}$ only if patient $>\mathbf{8 0} \mathbf{~ k g}$ & $37(52.9 \%)$ \\
\hline $\mathbf{N} / \mathbf{A}$ & $1(1.4 \%)$ \\
\hline
\end{tabular}

CI: 41, 64\%) responded that they prescribe two grams of Ancef per dose if the patient weighed greater than $80 \mathrm{~kg}$ (Table 4).

\section{Need for further research}

There was a considerable amount of support among respondents for further research including strong support for a large clinical trial to evaluate outcomes following different prophylactic antibiotic regimens. Specifically $84 \%$ (95\% CI: 73, 91\%) felt there was a need for further trials to evaluate outcomes following different prophylactic antibiotic drugs (Table 5). Eighty-three percent (95\% CI: 73, 91\%) felt there is a need for further trials to evaluate outcomes following different prophylactic antibiotic regimens (Table 5). An overwhelming 90\% (95\% CI: 81, 95\%) of respondents would change their practice if a large randomized controlled trial showed clear benefit of an antibiotic drug and regimen different from what they currently prescribe (Table 6) and 87\% (95\% CI: 77, 93\%) of respondents agreed they would participate in a large randomized control trial. The majority of respondents feel that as little as a $10 \%$ absolute risk reduction in infection would be a clinically significant benefit (Table 6).

\section{Discussion}

The results of this survey demonstrated five key findings. (1) Surgeons vary considerably in their choices of antibiotic regimens and dosages demonstrating a lack of consensus on which prophylactic antibiotic regimen is believed to be most effective. (2) The duration of prescribed regimen varies between surgeons as well with one in three surgeons believing antibiotics could be discontinued after 24 hours while $40 \%$ continue antibiotics until the suction drain is removed. (3) Surgeons also felt there was a lack of evidence and uncertainty with regard to which prescription of antibiotic regimen was most effective. (4) Respondents were overwhelmingly in favor of a large multi-center randomized control trial to assess the efficacy of different regimens. (5) More significantly ninety percent of surgeons agreed that they would change their practice if a large randomized controlled trial showed clear benefit of an antibiotic drug regimen different from what they are currently using with the majority of Orthopaedic Oncology Surgeons considering a 10\% absolute risk reduction in infection rates to be clinically significant. 
Table 5 Need for further research

\begin{tabular}{|c|c|c|c|c|c|}
\hline & Strongly agree & Agree & Unsure & Disagree & Strongly disagree \\
\hline $\begin{array}{l}\text { I feel there is a need for further trials to evaluate } \\
\text { outcomes following different prophylactic antibiotic drugs }\end{array}$ & $30(43.5 \%)$ & $28(40.6 \%)$ & $5(7.2 \%)$ & $3(4.3 \%)$ & $3(4.3 \%)$ \\
\hline $\begin{array}{l}\text { I feel there is a need for further trials to evaluate } \\
\text { outcomes following different prophylactic antibiotic regimens }\end{array}$ & $32(46.4 \%)$ & $26(37.7 \%)$ & $5(7.2 \%)$ & $4(5.8 \%)$ & $2(2.9 \%)$ \\
\hline $\begin{array}{l}\text { I feel there is a need for studies on the cost-effectiveness } \\
\text { of different antibiotic drugs and regimens }\end{array}$ & $18(26.1 \%)$ & $28(40.6 \%)$ & $11(15.9 \%)$ & $5(7.2 \%)$ & $7(10.1 \%)$ \\
\hline $\begin{array}{l}\text { I would change my practice if a large randomized } \\
\text { controlled trial showed clear benefit of an antibiotic drug } \\
\text { and regimen different from what I am currently using }\end{array}$ & $51(72.9 \%)$ & $12(17.1 \%)$ & $3(4.3 \%)$ & $1(1.4 \%)$ & $3(4.3 \%)$ \\
\hline
\end{tabular}

These results signify a significant lack of evidence and guidelines directing the prescription of prophylactic antibiotic regimens in musculoskeletal tumor surgery. With the emergence of resistant antimicrobial organisms and outbreaks of clostridium difficile in healthcare facilities, duration and prescription of antibiotics has proven to be an important clinical entity. At the same time, a high infection rate of $15-30 \%$ reported in many studies [2-6] highlights the importance of optimizing antibiotic regimens. There is no doubt that the development of clinical guidelines is of paramount and immediate importance.

Due to the fact that bone sarcomas are rare, a randomized clinical trial designed to create high level evidence would require multi-institutional and likely international participation. To date, this type of study has not been attempted in the Orthopaedic Oncology community. However, such a study is possible with support from a Surgical Trials Methods Center which exists at the institution of the primary authors for this study. A trial has been designed and is under funding and ethics review, which will involve randomizing patients undergoing lower extremity tumor prosthesis reconstruction to either 24 hours or 5 days of cephalosporin coverage. The study will be double-blinded as randomization will be completed by the Pharmacy Department at each institution. Thus, with completion of this study, there is the possibility that eventually guidelines such as those provided by the AAOS will be created for this very challenging peri-operative issue in Orthopaedic Oncology.

The strengths of our study include obtaining a comprehensive sampling of North American orthopaedic oncology surgeons from both the Canadian Orthopaedic Oncology Society (CANOOS) and Musculoskeletal Tumor Society (MSTS), achieving an exceptional survey response rate of approximately $75 \%$ that helps to limit non-responder bias with active surgeon participation along with a comprehensive sampling of surgeons from academic and nonacademic centres,

Our response rate of $75 \%$ of Orthoepdic Oncologists provided a robust data set for the general purposes of our study as well as exceeded the level for our anticipated study precision. Nevertheless, future studies that are aimed at more rigorously evaluating potential samplingbias will include surgeons from Europe and Asia. While non-responeder bias could not be eliminated it was minimized through re-administering the survey specifically to

Table 6 Clinical importance and interest in participating study

\begin{tabular}{|c|c|c|c|}
\hline & & No. & \\
\hline & Any reduction at all & 15 & $(21.7 \%)$ \\
\hline \multirow{10}{*}{$\begin{array}{l}\text { Amount an alternative antibiotic drug } \\
\text { and regimen needs to reduce infection } \\
\text { rate before the improvement is considered } \\
\text { "clinically important" }\end{array}$} & $5 \%$ & 10 & $(14.5 \%)$ \\
\hline & $10 \%$ & 15 & $(21.7 \%)$ \\
\hline & $15 \%$ & 4 & $(5.8 \%)$ \\
\hline & $20 \%$ & 12 & $(17.4 \%)$ \\
\hline & $25 \%$ & 8 & $(11.6 \%)$ \\
\hline & $30 \%$ & 1 & $(1.4 \%)$ \\
\hline & $35 \%$ & 0 & $(0.0 \%)$ \\
\hline & $40 \%$ & 0 & $(0.0 \%)$ \\
\hline & $50 \%$ & 2 & $(2.9 \%)$ \\
\hline & $>50 \%$ & 2 & $(2.9 \%)$ \\
\hline \multirow{2}{*}{$\begin{array}{l}\text { I would participate in a multi-centre } \\
\text { randomized controlled study assessing } \\
\text { different antibiotic regimens in long-bone } \\
\text { reconstruction for tumor surgery. }\end{array}$} & Yes & 61 & $(87.1 \%)$ \\
\hline & $\mathrm{No}$ & 9 & $(12.9 \%)$ \\
\hline
\end{tabular}


those who had yet to complete the survey.

\section{Conclusion}

We have shown that there are varied opinions and variations from surgeons on the prescription of prophylactic antibiotic regimens in tumor surgery. There is as yet no clinical trial on the efficacy of different antibiotic regimens in preventing infection in long-bone prosthetic reconstruction. However, there is overwhelming support in the Orthopaedic Oncology community to participate in a multi-center trial, which is currently in the state of development.

\section{Competing interests}

The author(s) declare that they have no competing of interest.

\section{Acknowledgements}

None to declare

\section{Author details}

${ }^{1}$ Department of Surgery, McMaster University, Hamilton, ON, Canada.

${ }^{2}$ Department of Surgery Surgery, University of Toronto, Toronto, ON, Canada. ${ }^{3}$ Department of Orthopedic Surgery, Vanderbilt University, Nashville, TN, USA. ${ }^{4}$ Department of Clinical Epidemiology and Biostatistics, McMaster University, Hamilton, ON, Canada.

\section{Authors' contributions}

$\mathrm{KH}$ carried assisted in the conception and design of the survey as well as data acquisition. $\mathrm{KH}$ assisted in analyzing and interpreting the data as well as drafting and revising the final manuscript. AR assisted in developing the figures and participated in revising the manuscript. BD assisted in study design, providing background knowledge as well as revising the manuscript. FF assisted in data analysis and interpretation. JW, PF, BP, and MB provided critical revisions to the manuscript as well as assisting in developing study design. MG conceived and designed the survey and the study. MG provided critical revisions and assisted in drafting the manuscript as well as approving the final manuscript for submission for publication.

Received: 15 July 2011 Accepted: 7 June 2012

Published: 7 June 2012

\section{References}

1. Morii $T$, Yabe H, Morioka H, Beppu Y, Chuman H, Kawai A, Takeda K, Kikuta K, Hosaka S, Yazawa Y, Takeuchi K, Anazawa U, Mochizuki K, Satomi K: Postoperative deep infection in tumor endoprosthesis reconstruction around the knee. EMBASE Journal of Orthopaedic Science 2010, 15(3):331-339.

2. The Paediatric Orthopaedic Society of North America (POSNA) http://www posna.org/education/studyguide/ewingssarcoma.asp

3. Jeys LM, Grimer RJ, Carter SR, Tillman RM: Periprosthetic infection in patients treated for an orthopaedic oncological condition. EMBASE Journal of Bone and Joint Surgery - Series A 2005, 87(4):842-849.

4. Jeys $L$, Grimer R: The long-term risks of infection and amputation with limb salvage surgery using endoprostheses. Recent Results Cancer Res. 2009, 179:75-84.

5. Jeys LM, Grimer RJ, Carter SR, Tillman RM: Periprosthetic infection in patients treated for an orthopaedic oncological condition. J Bone Joint Surg Am. 2005, 87(4):842-9.

6. Gaur AH, Liu T, Knapp KM, Daw NC, Rao BN, Neel MD, Rodriguez-Galindo C, Brand D, Adderson EE: Infections in children and young adults with bone malignancies undergoing limb-sparing surgery. EMBASE Cancer 2005, 104 (3):602-610.

7. Shehadeh A, Noveau J, Malawer M, Henshaw R: Late complications and survival of endoprosthetic reconstruction after resection of bone tumors. Clin Orthop Relat Res. 2010, 468(11):2885-95.

8. Jeys LM, Grimer RJ, Carter SR, Tillman RM: Risk of amputation following limb salvage surgery withendoprosthetic replacement, in a consecutive series of 1261 patients. Int Orthop 2003, 27(3):160-3. Epub 2003Feb 8.
9. Grimer RJ, Belthur M, Chandrasekar C, Carter SR, Tillman RM: Two-Stage Revision for Infected Endoprostheses Used in Tumor Surgery. Clinical Orthopaedics \& Related Research 2002, 395:193-203. SECTION II ORIGINAL ARTICLES: Tumor.

10. Ward WG, Johnston-Jones K, Lowenbraun S, Dorey F, Rosen G, Eckardt JJ: Antibiotic prophylaxis and infection resistance of massive tumor endoprostheses during chemotherapy. EMBASE Journal of the Southern Orthopaedic Association 1997, 6(3):180-185. Fall 1997.

11. AlBuhairan B, Hind D, Hutchinson A. Antibiotic prophylaxis for wound infections in total joint arthroplasty: a systematic review. J Bone Joint Surg Br. 2008 Jul; 90(7):915-9. Clinical Trials Research Unit, School of Health and Related Research, University of Sheffield, Regent Court, 30 Regent Street, Sheffield S1 4DA.

12. Costelloe C, Metcalfe C, Lovering A, Mant D, Hay AD. Effect of antibiotic prescribing in primary care on antimicrobial resistance in individual patients: systematic review and meta-analysis. BMJ. 2010 May 18; 340:c2096. doi: 10.1136/bmj.c2096. Academic Unit of Primary Health Care, NIHR National School for Primary Care Research, Department of Community Based Medicine, University of Bristol, Bristol BS8 2AA

13. Griffith L, Cook DJ, Guyatt GH, Charles CA: Comparison of open and closed questionnaire formats in obtaining demographic information from Canadian general internists. Journal of Clinical Epidemiology 1999 Oct; 52(10):997-1005. Department of Clinical Epidemiology Biostatistics, McMaster University Faculty of Health Sciences, Hamilton, Ontario, Canada.

14. Armstrong D, Ashworth M: When questionnaire response rates do matter: a survey of general practitioners and their views of NHS changes. British Journal of General Practice 2000 Jun, 50(455):479-480.

15. Templeton L, Deehan A, Taylor C, Drummond C, Strang J: Surveying general practitioners:does a low response rate matter? British Journal of General Practice 1997, 47:91-94.

doi:10.1186/1471-2474-13-91

Cite this article as: Hasan et al:: Prophylactic antibiotic regimens in tumor surgery (PARITY) survey. BMC Musculoskeletal Disorders 2012 13:91.

\section{Submit your next manuscript to BioMed Central and take full advantage of:}

- Convenient online submission

- Thorough peer review

- No space constraints or color figure charges

- Immediate publication on acceptance

- Inclusion in PubMed, CAS, Scopus and Google Scholar

- Research which is freely available for redistribution 